\title{
XIV. Two new pressure-gauges for the highest vacua
}

\section{William Sutherland}

To cite this article: William Sutherland (1897) XIV. Two new pressure-gauges for the highest vacua, Philosophical Magazine Series 5, 43:261, 83-99, DOI: $10.1080 / 14786449708620965$

To link to this article: http://dx.doi.org/10.1080/14786449708620965

曲 Published online: 08 May 2009.

Submit your article to this journal $\pi$

Џ Article views: 7

Q View related articles $\longleftarrow$ 
for its discharges, as we have pointed out, are free from the fluctuating effects produced by induction-coils, transformers, and electrical machines. Our present paper is therefore only preliminary to a more exhaustive study of the discharges of electricity through rarefied gases by means of a storage battery of ten thousand cells, which will give an electromotive force of about twenty thousand volts.

Harvard University, Cambridge, Mass., U.S.A., December 1st, 189:.

XIV. Two New Pressure-Gauges for the Highest Vacua. By William Sutherland *

TITHERTO the $\mathbb{M}^{\circ}$ Leod gauge has been accepted as the most accurate instrument for the measurement of pressures in vacua, although there has been a good deal of misgiving as to the limit up to which its indications are fairly reliable, with an impression that near a millionth of an atmo its measurements become illusory. Baly and Ramsay, in their experiments on rare gases (Phil. Mag. [5] xxxviii.), after working with two M'Leod gauges of very high sensitiveness, pronounced the type of gauge worthless for air and $\mathrm{CO}_{2}$ though reliable for hydrogen ; but the failure of their gauges with air was due to a most interesting and very special phenomenon, to bo considered in my next paper, liable to occur only under special circumstances and not affecting the principle of the gauge or its application to air with avoidance of the perturbing conditions. In the case of $\mathrm{CO}_{2}$ the $\mathrm{M}^{\prime}$ Leod gauge is less reliable than with other gases on account of some action between glass and $\mathrm{CO}_{2}$, especially if moisture be present; but this does not necessitate that the gauge should be worthless for $\mathrm{CO}_{2}$, but that there should be a limit to its application, this limit probably depending on the nature of the glass and its treatment. Towards the end of this paper it will be shown that Crookes's $M^{\circ}$ Leod gauge begins to fail decidedly at $5 / 10^{6}$ atmo, with increasing unreliability at lower pressures, the cause of which will be proved to be probably a small residue of water-vapour dissolved, so to speak, in the glass of the gauge. Thus, if the theoretical reasoning of "Buyle's Law at very Low Pressures" + is sound, the principle of the $M^{\prime}$ 'Leod gauge ought to be applicable up to any degree of rarefaction, if we can only make it of such a material or so manipulate it as to free it from this trouble with moisture.

* Communicated by the Author.

$\dagger$ Suprà, p. 11. 
But it is very desirable to have an independent method and instrument for controlling the indications of the $M^{\circ}$ Leod gauge, reaching to higher vacua and simpler in its practical working; and one outcome of the paper on "Thermal Transpiration and Radiometer Motion"* is the furnishing of two instruments capable of independently measuring the pressure in high vacua. These two instruments were in a manner united into a single one in Crookes's torsion radiometer briefly described but rather fully discussed in that paper; and though for some purposes there might be advantage in uniting the two forms, each would be capable of its best performance when separate. The two instruments are a disk viscosity-meter and a torsional radiometer of special design to be discussed in this paper. Crookes used his torsion radiometer as a viscosity-meter by forcing it to vibrate and determining the decrement of the logarithm of the angular amplitude of successive vibrations. At pressures near one atmo the vertical plate of the radiometer in oscillating round a vertical axis in the bulb sweeps air bodily in front of it and draws air behind it in a very complex manner, so that its motion is conditioned partly by the mass motion of the gas, as well as by viscosity of gas and viscosity of suspending fibre; but as Crookes's experiments showed, in a manner brought out by Stokes's theoretical discussion (Phil. Trans. clxxii.), the effect of the mass motion of the gas on the log. dec. disappears rapidly, and the log. dec. is practically constant for a considerable range of pressure, proving that viscosity alone is effective in retarding the motion of the vibrating vertical plate. The following values of $10^{4}$ times the log. dec. in air are taken from Crookes's results (Phil. Trans. clxxii.) :-

$$
\begin{array}{rrrrrrrrrr}
p \text { (mm.) } \ldots & 760 & 550 & 301 & 155 & 47 & 12 & 4 & 3 & 1 \\
& 1124 & 1073 & 1022 & 1006 & 1001 & 1000 & 1000 & 994 & 988
\end{array}
$$

These show that from $47 \mathrm{~mm}$. to $4 \mathrm{~mm}$, the latter being only $1 / 12$ of the former, the log. dec. is constant; so that at this range of pressure the mass motion of the gas is negligible, and the viscosity is independent of pressure, as it ought to be according to Maxwell's discovery, so long as slipping of the gas on the solid surfaces of the edge of the plate and the walls of the bulb is negligible; but then at $3 \mathrm{~mm}$. and $1 \mathrm{~mm}$. the log. dec. diminishes decidedly in a manner more fully illustrated by the following further data from Crookes, the unit of pressure being now $1 / 10^{6}$ atmo :-

$$
\begin{array}{rrrrrrrrr}
p \ldots \ldots . . . . & 1000 & 495 & 300 & 100 & 53 & 24 & 13 & 8 \\
988 & 966 & 952 & 876 & 774 & 620 & 500 & 390 \\
\text { * Phil. Mag. for November and December } 1896 . &
\end{array}
$$


Now, as was first shown by Kundt and Warburg, and as has been shown in "Thermal Transpiration and Radiometer Motion," the effect of slipping of gas on the influence of viscosity is to replace the viscosity $\eta$ by $\eta /(1+2 \zeta / D)$, where $\zeta$ is the coefficient of slipping and equal to $a \lambda_{s}$, where $a$ is a fraction which is probably nearly the same for most gases, and $\lambda_{s}$ is the mean free path of a molecule of the gas near the solid surface, $\mathrm{D}$ being distance between moving and resting solid surfaces; for a given gas, $\lambda_{s}$ is nearly proportional to $\lambda$, the mean free path of a molecule far from the surface, and as $\lambda$ is inversely proportional to the density, we have the result that $\zeta$ varies inversely as the density of the gas. Thus a measurement of slipping furnishes a means of measuring density, and therefore, if the law connecting density and pressure is known, of measuring pressure.

If Boyle's law holds, then $\lambda=\lambda_{0} p_{v} / p$, where $\lambda_{0}$ is the mean free path at some stindard pressure $\mu_{0}$; thus $\eta$ is replaced by $\eta /\left(1+2 a \lambda_{0} p_{0} / \mathrm{D} p\right)$. When $p$ is large enough, this is indistinguishable from $\eta$, as we saw to be the case in Crookes's experiments with air from $47 \mathrm{~mm}$. to $4 \mathrm{~mm}$., through which range the log. dec. retained a constant value which we may denote by L. Then, if $l$ is the log. dec. at lower pressures, $\mathrm{L} / l$ gives $1+2 a \lambda_{0} p_{0} / \mathrm{D} p$, whence we can get values of $2 a \lambda_{0} p_{0} / \mathrm{D}$ which ought to be all the same. But there is first one little correction to make, namely that for the viscosity of the torsion fibre, because a small constant portion of the log. dec. is due to its small viscosity ; it is the value of the log. dec. for the apparatus if an absolute vacuum were attained in it. Call this part of the log. dec. $\mu$, then

$$
\left(\frac{\mathrm{L}-\mu}{l-\mu}-1\right) p=2 a \lambda_{0} p_{0} / \mathrm{D} . \quad \text {. . . }
$$

At the higher values of $p$ we can neglect $\mu$ and obtain at once a mean value of the constant $2 a \lambda_{0} p_{0} / \mathrm{D}$, and then with this as a known quantity solve for $\mu$ at the lower pressures. In this way, from the data already given for air, $2 a \lambda_{0} p_{0} / \mathrm{D}$ appears to be 15 and $\mu$ to be $\cdot 004$. With these values and that for $\mathrm{L}$, namely $\cdot 1000$, the last equation becomes one for obtaining $p$ by a measurement of $l$; and to show how it works, we give the values of $p$ obtained by it from Crookes's values of $l$ already given :-

$\begin{array}{lllllllll}p \text { by gauge ........... } & 1000 & 495 & 300 & 100 & \mathbf{5 3} & 24 & 13 & 8 \\ p \text { from log. dec. ...... } & 1153 & 406 & 283 & 101 & \mathbf{4 9} & 23 & 13 \cdot 8 & 8 \cdot 6\end{array}$

Now it is obvious that the new method of finding the 
pressure in a vacuum becomes less delicate when $l$ becomes nearly equal to $\mathrm{L}$, for then the value of $p$ is liable to be greatly affected by only a small error in $l$. Thus, for example, a diminution of $l$ in the first case from $\cdot 0988$ to $\cdot 0986$ would reduce the calculated pressure to 1000 ; thus the first two or three values of the calculated pressures belong to a range where this particular torsion radiometer is not sensitive as a pressure-gauge. Of course we are at present assuming that the pressures as given by Crookes's $\mathbf{M}^{\prime}$ Leod gauge are correct; it must be remembered that what the indications of the M'Leod gauge really amount to is a sort of a measure of the density of the gas, with an inference as to its pressure by means of Boyle's law, and I have already pointed out that the measurement deduced from slipping is strictly only a measurement of density with a pressure inferred by means of Boyle's law ; the fact, therefore, that the pressures as found by the log. dec. and by the $M^{\prime}$ Leod gauge in the last table are on the whole so consistent furnishes no proof of the validity of Boyle's law at low pressures, but this, however, has been fairly established on other grounds in "Boyle's Law at very Low Pressures," and therefore the consistency of the two sets of pressures is a proof of the relative correctness of the pressures obtained by Crookes with his M'Leod gauge.

The great advantage about the new viscosity-meter gauge is that its sensitiveness tends to increase with diminution of the pressure which it has to measure. Let us therefore proceed to compare the pressures of air as found by Crookes's M'Leod gauge at still lower pressures, and as calculated by (34) from his measurements of the log. dec.

\begin{tabular}{|c|c|c|c|c|c|c|}
\hline $\begin{array}{ll}\ldots \ldots \ldots & 7 \cdot 2\end{array}$ & 5.9 & $4 \cdot 1$ & $3 \cdot 4$ & $2 \cdot 6$ & $1 \cdot 9$ & $1 \cdot 3$ \\
\hline$p$ from log. dec. $\quad \ldots \quad 7.9$ & 6.7 & $5 \cdot 0$ & $4 \cdot 4$ & $3 \cdot 6$ & 3.0 & $2 \cdot 5$ \\
\hline $10^{4}$ log. dec. ....... 372 & 337 & 281 & 256 & 225 & 198 & 175 \\
\hline$p$ by gauge....... & .55 & -46 & 22 & $\cdot 14$ & $\cdot 06$ & 0 \\
\hline$p$ from log. dec. ... & $1 \cdot 82$ & $1 \cdot 65$ & $1 \cdot 33$ & 1.25 & .95 & $\cdot 5$ \\
\hline $10^{4}$ log. dec.......... & 144 & 135 & 118 & 114 & 97 & 7 \\
\hline
\end{tabular}

Here we have increasing divergence between the two values of the pressure, till at a pressure estimated as $\cdot 02 / 10^{6}$ atmo by the M'Leod gauge and Boyle's law the log. dec. in the viscosity-meter gives a pressure 26 times as great. Now it is rather important that we should have some test as to which of the above series of pressures is the more reliable, because although from what we know of the tenacious eling of $\mathrm{H}_{2} \mathrm{O}$ to glass there is reason to expect the M'Leod gauge to fail at some point, still we cannot without further support attribute 
the discrepancy in the last comparison entirely to the inadequacy of the indications of the gauge at these very low pressures.

In Crookes's measurements of the apparent repulsive force of a candle on his torsion radiometer we have another means of determining the pressure independently, and the torsion radiometer thus used becomes our second form of pressuregauge. In "Thermal 'Transpiration and Radiometer Motion" it was shown that the deflecting force exercised by a source of constant radiation on the vertical plate of the torsion radiometer is connected with the pressure of the gas by the formula

$$
\text { deflecting force }=c^{\prime} /\left(\mathrm{A}^{\prime \prime} p+\mathrm{B}^{\prime \prime \prime}+1 / p\right) . . .
$$

But we must remember that this is derived from the equation (12),

$$
\frac{d p}{d x} \cdot \frac{3 \mathrm{R}^{2}}{4 \eta}(1+4 \zeta / \mathrm{R})=-v \lambda\left(\frac{1}{n} \frac{d p}{d x}+\frac{1}{v} \frac{d v}{d x}\right)
$$

which, when $n$ is large enough, reduces to

$$
\frac{d p}{d x} \frac{3 \mathrm{R}^{2}}{4 \eta}=-\lambda \frac{d v}{d x}
$$

whence the deflecting force becomes proportional to $\lambda$-that is, inversely proportional to the density. Hence, at a large enough value of $n$, the deflecting force gives a measure only of the density, just as the M'Leod gauge and the viscositymeter gauge do ; and it is only by using Boyle's law that we get the form of equation (26) given above, which, when $p$ is large enough, becomes

$$
\text { deflecting force }=c^{\prime} / \mathrm{A}^{\prime \prime} p \text {. }
$$

Similarly it can be shown that when $p$ becomes small enough the equation really only gives a connexion between the mean density and the difference of density at the front and back of the plate, which passes into a connexion between deflecting force and pressure only on the assumption of a law connecting density and pressure. When this is Boyle's law, the complete equation is (26) above. For Crookes's instrument with air $c^{\prime}=3 \cdot 0, \mathrm{~A}^{\prime \prime}=\cdot 001$, and $\mathrm{B}^{\prime \prime \prime}=\cdot 01$ when the unit of pressure is $1 / 10^{6}$ atmo and the unit of deflecting force arbitrary. Accordingly, with the values of the deflecting force at low pressure, we can calculate the pressures; thus in the following table we give the pressure as given by the M'Leod gauge with Boyle's law, by the deflecting force with equation (26), and by the log. dec. as given in the last table, and we add a row 
for the ratio of pressure from log. dec. to pressure from deflecting force.

\begin{tabular}{|c|c|c|c|c|c|c|c|c|c|}
\hline Def'. for............. & $30 \cdot 9$ & & 23 & $20 \cdot 2$ & 1 & & $13 \cdot 1$ & $11 \cdot 5$ & 8.5 \\
\hline$p$ by gauge....... & 13 & & 8 & $7 \cdot 2$ & 5 & & $4 \cdot 1$ & $3 \cdot 4$ & $2 \cdot 6$ \\
\hline$p$ from def. for.... & $13 \cdot 5$ & & $8 \cdot 6$ & $7 \cdot 6$ & 6 & & $4 \cdot 7$ & $4 \cdot 0$ & \\
\hline \multirow[t]{2}{*}{$p$ from log. dec.... } & $13 \cdot 8$ & & $8 \cdot 6$ & $7 \cdot 9$ & $6^{\circ}$ & & $5 \cdot 0$ & $4 \cdot 4$ & \\
\hline & $1 \cdot 0$ & & $1 \cdot 0$ & 10 & 1 . & & $1 \cdot 1$ & $1 \cdot 1$ & \\
\hline Def. for. ............ & $7 \cdot 1$ & $4 \cdot 2$ & $2 \cdot 1$ & $2 \cdot 0$ & 17 & $1 \cdot 4$ & $1 \cdot 0$ & 7 & $\cdot 5$ \\
\hline$p$ by gauge ...... , & $1 \cdot 9$ & $1 \cdot 3$ & $1 \cdot 0$ & .55 & -46 & $\cdot 22$ & $\cdot 14$ & $\cdot 06$ & .02 \\
\hline$p$ from def. for. $\ldots$ & 24 & $1 \cdot 4$ & 70 & 67 & 57 & $\cdot 47$ & $\cdot 33$ & $\cdot 23$ & $\cdot 17$ \\
\hline \multirow[t]{2}{*}{$p$ from $\log$. dec. ... } & $3 \cdot 0$ & 25 & $2 \cdot 2$ & 18 & 165 & 1.33 & $1 \cdot 25$ & $\cdot 95$ & .53 \\
\hline & 12 & 1.8 & $3 \cdot 1$ & 27 & 29 & 28 & $3 \cdot 8$ & $4 \cdot 1$ & 31 \\
\hline
\end{tabular}

At first sight these numbers seem to show that the pressures given by the deflecting force are in much better harmony with those given by the gauge than are those given by log. dec., but we shall show that this is due to a defect in Crookes's torsion radiometer when made to do duty as a pressure-gauge, a defect which could be avoided in a suitable design; for a study of the row of ratios shows that while the value increases slowly from $1 \cdot 0$ to $1 \cdot 2$ there is a sudden transition to a value about $3 \% 0$, and the theoretical reason for expecting such a change of ratio was pointed out in "Thermal Transpiration and Radiometer Motion," as it was stated that in Crookes's instrument the formula would represent the relation between deflecting force and pressure as long as the vertical plate was able to control the fall of temperature in the gas near its edge, but that it would cease to do this as soon as the mean path of the molecules became nearly equal to the radius of the bulb, for then the great surface of the bulb compared to the thickness of the plate would enable it to swamp the influence of the plate, even in the plate's own neighbourhood; thus there should be a change from the limiting relation def. force $=c^{\prime} p$ for the plate, to def. force $=c^{\prime \prime} p$ for the bulb. Now the above change of ratio is established at a pressure about $2 \cdot 5 / 10^{6}$ atmo as given by log. dec., when the mean free path of a molecule of air ought to be about $10^{6} \times \cdot 00001 \div 2.5$ centim. or 4 centim., which is about the radius of the bulb. The approximate constancy of the new ratio at about 3 should increase our confidence in the pressures derived from the log. dec. as continuously fairly reliable down to the lowest pressure of half-a-millionth of an atmo, whereas the M'Leod gauge gives pressures only half as large as they ought to be at about $2 / 10^{6}$ atmo and about $1 / 26$ as large as they ought to be at $5 / 10^{6}$ atmo. 
Thus Crookes's data for air show the principles of both the new gauges to be sound, but to get the best results from each it ought to be developed on its own lines. To avoid prolixity we will call the viscosity-meter when used as a rressure-gauge the Viscometer Gauge; its design on the large seale has been worked out by Maxwell (Phil. Trans. 1866), who also furnished the theory of it, and on a smaller scale by Kundt and Warburg (Pogg. Ann. elvi.). A still sinaller scale would, in most cases, be advisable for the viscometer gauge, as its volume ought to be kept small. In Maxwell's apparatus three glass plates 28 centim. in diameter and 2 millim. thick were fixed at equal distances along a solid axis, the continuation of which was a fine vertical torsion-wire of steel ; fixed disks were mounted so that each of the movable disks was half-way between two fixed ones at distances varying from 5 millim. to 17 ; the whole was enclosed in a glass vessel with a vertical continuation for the 125 centim. of torsion-wire. The inconvenience about this form is that special arrangements bave to be made for determining the viscosity of the rather strong wire that is required to support so heavy a system. Kundt and Warburg simplified the apparatus by reducing the weight and adopting a bifilar suspension whose viscosity effect was practically negligible. They used a single movable plate of glass of 16 centim. diameter and thickness about 1 millim. vibrating between two other fixed ones, the distance between fixed and moving surfaces varying from $1 \cdot 1$ millim. to $2 \cdot 8$. It is to be remembered that both these instruments were designed to obtain accurate absolute values of the coefficients of viscosity of gases, and for that purpose Maxwell worked out a pretty complete theoretical expression connecting decrement of the logarithm of consecutive angular amplitudes of the vibrating system with the viscosity of the gas between the plates, the viscosity of the wire, and the constants of the apparatus.

Now we have just seen that Crookes succeeded in getting remarkably good readings of log. dec. with his apparatus, where the surface at which the viscosity produces its effect is only the minute thickness of a small straight edge of a mica plate moving not particularly close to a spherical surface, that is to say, under very unfavourable circumstances; it is therefore evident that a thin glass, mica, or untarnishable metal disk about 5 centim. in diameter and as light as possible, vibrating between two fixed plates with a distance not greater than about 1 millim. between fixed and moving surfaces, the suspension being by means of a quartz thread, would give values of $\log$. dec. of far higher accuracy than 
Crookes's, and would therefore enable our formula to give the coefficient of slipping $\zeta$ with great accuracy, and conversely the pressure.

The simplest form which the apparatus could take would be that in which the two fixed plates form the top and bottom of a shallow cylindrical drum strong enough to stand a pressure of an atmo on its outer wall without appreciable deformation; within the drum the movable disk is suspended equidistant from top and bottom by a quartz fibre passing down the axis of a fine vertical tube rising from the centre of the top of the drum; a small tube in the centre of the bottom would furnish connexion with the gas supply whose pressure is to be measured, a side tube from the upper vertical tube facilitating the operation of filling the drum with the pure gas. A small mirror attached to the quartz fibre at a suitable point near its entrànce to the drum would give the means of measuring the angular amplitude of successive vibrations of the disk, which could be set in motion by means of a small magnet attached to the back of the mirror.

The whole apparatus ought to be made so that it could be raised to as high a temperature as possible to secure thorough drying and exhaustion. The special advantage of this form of viscometer gauge is that its volume is small, only the moccupied space of the drum, say $2 \pi \times 2 \cdot 5^{2} \times 1$ cub. centim., 4 gether with the internal volume of the tube, which is, say, 50 centim. long and 1 millim. radius, namely $\pi \times 1^{2} \times 50$ cub. centim., that is a total volume of about 6 cub. centim.

A more accurately constructable form of the viscometer gauge would be one in which the two fixed disks and the movable one with its suspension fibre passing through a hole in the upper are all enclosed as in Maxwell's and in Kundt and Warburg's apparatus in a suitable vessel for connexion with the gas supply whose pressure is to be measured. Now in these forms of viscometer gauge the expression given by Maxwell for the relation between log. dec. and viscosity simplifies at, say, 1/20 atmo to one which makes the log. dec. minus the unknown log. dec. of an absolute vacuum due to the viscosity of the fibre, proportional to the viscosity of the gas. The part of the log. dec. due to the fibre could be found by loading the fibre with a sphere of lead equal in weight to the disk of the viscometer gauge and observing the log. dec. in air at any ordinary pressure, the small effect of the viscosity of the air being allowed for by calculation, or the sphere might be torsionally vibrated in a bulb exhausted till the highest obtainable vacuum was reached, when the log. dec. ought to become constant or tend to a constant limit 
which could be definitely inferred, and would give the part to be subtracted from all readings of the log. dec. in the viscometer gauge as due to the fibre. Or $\mu$ in our formula (34) could be found directly from the readings of the values of the log. dec. as we found it with Crookes's observations, that is to say, by making observations of the log. dec. at different pressures measured by a reliable form of $\mathbf{M}$ 'Leod gauge. One measurement of $I_{\perp}$ and two measurements of $l$ at suitable pressures would suffice for the determination of $\mu$ and $2 a \lambda_{0} p_{0} / \mathrm{D}$, but mean values of these obtained from a number of observations would of course be preferable. The gauge is then ready for the measurement of any pressure in the particular gas under observation; for although $\mu$ is the same whatever the gas may be, $2 a \lambda_{0} p_{0} / \mathrm{D}$ varies from one gas to another ; but when once $\mu$ has been obtained it is only necessary to take a reading of $L$ and one reading of $l$ at any measured suitable pressure to give $2 a \lambda_{0} p_{0} / \mathrm{D}$ for the gas under study, and then all other pressures are obtainable by measurements of the $\log$. dec. and a simple calculation.

One advantage of the viscometer gauge is that when once its constants have been determined for any particular gas, the only operation necessary for obtaining any pressure is that of observing the amplitude of the $m$ th, $n$ th, and $p$ th vibrations, an operation so simple that it need only delay the experimenter a minute or two, and by a short calculation he can satisfy himself in a moment as to the magnitude of the pressure in his gas supply.

The other form of pressure-gange, the torsion radiometer, or, as we may call it, the Transpiration Gauge, bas already had its essential features indicated in "Thermal Transpiration and Radiometer Motion" as a piston enclosed in a cylinder with a uniform distance between the curved surfaces of both, the ends of the cylinder to be of glass or other transparent material to facilitate irradiation of one end of the piston by means of a constant source of heat such as a platinum spiral carrying a constant electric current or a steam bath; or the ends might be metal with a steam bath at one end and ice bath at the other. It has been shown in "Thermal Transpiration and Radiometer Motion" that when the temperatures of the two ends of the piston become constant there is a pressure driving the piston from hot to cold with a total force $c^{\prime} /\left(\mathrm{A}^{\prime \prime} p+\mathrm{B}^{\prime \prime \prime}+1 / p\right)$, where the coefficients are all constant for a given apparatus and a given gas, but varying with the gas. It is necessary to attach to the piston some means of enabling it to retain its position against this force, and of measuring the amount of the force; this may be done by 
placing the axis of cylinder and piston horizontal and in one of the lines where the horizontal plane through the axis cuts the cylinder, piercing it with a small hole at its middle point; this hole establishes communication with a small chamber, and through it passes an arm for supporting the piston in its right position, the chamber being large enough to hold a counterpoise to the piston attached to the other end of the arm; from the top of the chamber rises the fine vertical tube which is to contain the quartz torsion fibre rigidly attached to the arm and connected at the top of the tube with a torsion head, whereby a sufficient couple can be applied to balance the twisting effect of the pressure on the piston. In order not to interfere with the vacuum in the apparatus the torsion head should consist of a small magnet so pivoted that it can be turned through any angle by a large magnet outside, and so twist the quartz fibre through an angle measured by the usual mirror arrangement. To bring the piston always to the same position a mark on the counterpoise as seen through a window in the little chamber is brought into alignment with a mark on the window and a fixed point of reference such as cross fibres at a convenient position outside. The angle of torsion can be written equal to $e^{\prime} /\left(\mathrm{A}^{\prime \prime} p+\mathrm{B}^{\prime \prime \prime}+1 / p\right)$, and three or more measurements of its value at pressures determined by a $\mathbb{M}$ 'Leod gauge of assured reliability furnish the values of the constants or rather parameters $c^{\prime}, \mathrm{A}^{\prime \prime}, \mathrm{B}^{\prime \prime \prime}$ for the instrument and the gas, and then any other pressure can be calculated from an observation of the angle of torsion.

As to the material for the piston, Crookes's experience seems to show that a disk of mica blackened on one side would be eminently suitable; a circular disk, out of which a smaller one was cut, leaving an empty ring between the two, would form both piston and cylinder, and there would only remain to attach the solid ring inside the drum or vessel of suitable shape which is to hold the gas under study; for example, the solid ring could be cut of such a size that it just fits into a shallow cylinder to which it can be cemented, so that when the top and bottom of the cylinder are closed by thin glass or metal plates, and when the piston is in position, there are two shallow cylindrical chambers communicating through the annular space between piston and cylinder. The junctions would have to be such that the whole could be raised to $200^{\circ}$ or $300^{\circ} \mathrm{C}$. for thorough drying and evacuation. The front of both the disk and ring of mica being black and the back clear, there would be established a fall of temperature through the annular interspace such as is 
contemplated in "Thermal Transpiration and Radioneter Mation." For the highest accuracy it would be necessary to take steps to keep the whole drum at a constant mean temperature.

A special advantage of this form of gauge is that after a certain degree of rarefaction is reached $A^{\prime \prime} p+B^{\prime \prime \prime}$ may be neglected, and the deflecting force and angle of torsion become proportional to the pressure, so that the experimenter can ascertain at a glance what pressure he is dealing with.

To show a defect to which both new forms of gauge are subject, and yet how they can support one another and bring to light an interesting phenomenon, we will discuss Crookes's observations on hydrogen just as we have done those on air. In "Thermal 'Transpiration and Radiometer Motion" it was shown that the deflecting force for hydrogen is not expressed by the ordinary formula until changed to

$$
c^{\prime} /\left\{\mathrm{A}^{\prime \prime} p /(1-\alpha p)+\mathrm{B}^{\prime \prime \prime}+1 / p\right\} \text {. }
$$

Now the term $A^{\prime \prime} p$ in the ordinary formula originates from a term involving the product of the mean free path near the solid and the mean free path far from the solid: and the mean free path near the solid was shown to be altered by condensation of the gas, but in such a manner that it is always proportional to the free path in the gas far from the solid; thus molecular attraction of the solid for the gas alters slightly the numerical value of $\mathrm{A}^{\prime \prime}$, but does not introduce any factor $1 /(1-\alpha p)$; therefore the introduction of this factor is probably due to some other surface action; if it is really connected with some other alteration of the free path of a molecule of the gas near the solid surface we ought to find the slipping of the gas affeeted; for theoretically the amount of slipping is proportional to the mean free path near the solid, and at the same time, as the free path far from the surface is unaffected, the viscosity of the gas should be practically unaltered by the surface action. Accordingly some interest attaches to the study of slipping in Crookes's experiments with hydrogen. The constant upper limit for the log. dec. in $\mathrm{H}_{2}$ is $\cdot 0499$, and in the case of air we took $\mu$, the part of the log. dec. due to viscosity of fibre, as 0040 ; thus in (34) we have $\mathrm{L}$ and $\mu$, the values of $l$ being given in the next table at the pressures given as found by the $M^{6}$ Leod gauge and Boyle's law in terms of $1 / 10^{6}$ atmo as unit; in the third row will be found $(\mathrm{L}-\mu) /(l-\mu)-1$, and in the fourth

$$
\{(\mathrm{L}-\mu) /(l-\mu)-1\} p,
$$

Phil. Mag. S. 5. Vol. 43. No. 261. Feb, 1897. 
which, under ordinary circumstances, ought to have the constant value $2 a \lambda_{0} p_{0} / \mathrm{D}$.

$\begin{array}{rrrrrrrrrrr}p \text { by gauge... } & 330 & 234 & 155 & 110 & 59 & 37 & 26 \cdot 5 & 20 & 14 \cdot 5 & 8 \\ 10^{4} \text { log. dec.. } & 495 & 488 & 484 & 472 & 441 & 408 & 373 & 351 & 324 & 270 \\ & \cdot 009 & 024 & \cdot 034 & -063 & \cdot 145 & \cdot 247 & -378 & \cdot 476 & \cdot 616 & \cdot 995 \\ & 3 \cdot 0 & 5 \cdot 6 & 5 \cdot 3 & 6 \cdot 9 & 8 \cdot 6 & 9 \cdot 0 & 10 \cdot 0 & 9 \cdot 5 & 8 \cdot 9 & 8 \cdot 0 \\ 12 \cdot 9 & 12 \cdot 6 & 9 \cdot 9 & 10 \cdot 2 & 10 \cdot 3 & 10 \cdot 2 & 10 \cdot 8 & 10 \cdot 1 & 9 \cdot 3 & 8 \cdot 2\end{array}$

The numbers in the fourth row instead of being constant rise to a maximum and decline; but if to the numbers in the third row, which represent $2 \xi / \mathrm{D}$, we add $\cdot 03$ and then multiply by $p$, we get the products given in the last row, which are practically constant down to $20 / 10^{6}$ atmo. Thus, then, the free path near the solid $\lambda_{s}$, which is proportional to the coefficient of slipping, instead of being proportional to $\lambda$ is proportional to $\lambda-h$, where $h$ is a constant.

Now in the formula for deflecting force $\mathrm{A}^{\prime \prime} p$ is the form given to a term varying inversely as $\lambda_{s}$, so that this term cannot be written as inversely proportional to $\lambda$, and therefore directly proportional to $p$, but it must be taken as inversely proportional to $\lambda(1-h / \lambda)$, that is to $\left(1-p h \lambda_{0} p_{0}\right) / p$, and therefore the term from which $A^{\prime \prime} p$ is derived in the general theory gives $\mathrm{A}^{\prime \prime} p /\left(1-p h / \lambda_{0} p_{0}\right)$ in the case of hydrogen, which, in "Thermal Transpiration and Radiometer" Motion," we actually found to be the case, if we identify $h / \lambda_{0} p_{0}$ with the constant $\alpha$ of that paper. And there we also found the value of $\mathrm{A}^{\prime \prime}$ to take its theoretical place along with those for other gases, which shows that in connexion with the deflecting force $\lambda_{s}$ in hydrogen is altered in its relation to $\lambda$ simply by a factor such as $\left(1-p h / \lambda_{0} p_{0}\right)$ with a limit 1 and not any other number; but. if from the last table we take 10 as the value of $\left(2 \xi / \mathrm{D}+{ }^{\circ} 03\right) p$, and compare it with the value 15 for $(2 \zeta / D) p$ in air, which stands for $2 a \lambda_{0} p_{0} / \mathrm{D}$, and remember that at any standard pressure $p_{0}$ the values of $\lambda_{0}$ for bydrogen and air are as those of $\eta / m^{\frac{1}{2}}$, that is nearly as 2 to 1 , we find that $(2 \zeta / \mathrm{D}+\cdot 03) p$ for hydrogen would have to become 30 to give this theoretical relation of 2 to 1 if the alteration of $\cdot 03$ were all that is required; this then indicates that with hydrogen, in addition to a factor $\left(1-p h / \lambda_{0} p_{0}\right)$ in the relation of $\lambda_{s}$ to $\lambda$, there is a factor $1 / 3$ whose occurrence here, and not in deflecting force, has to be explained.

The explanation probably lies in the presence of a trace of water-vapour, for notwithstanding the very thorough measures which Crookes took to get rid of the water-vapour after it had given him considerable trouble, I am still in- 
clined to think that a trace had been left in the hydrogen which both the Viscometer Gauge and the Transpiration Gauge have succeeded in detecting. The first question that naturally arises is, why should we not have similar evidence of the presence of water-vapour in the case of other gases? the answer to which is that, just as Graham showed experimentally in his study of the viscosity of mixed gases that the viscosity of hydrogen is specially sensitive to the presence of other gases, a partial theory of the phenomenon being given in "The Viscosity of Mixed Gases" (Phil. Mag. [5] xl.), so it is probable that a small amount of impurity largely affects the slipping of hydrogen while only slightly affecting that of the other gases, the difference between the case of hydrogen and those of the other gases being due to two causes, first, the small attraction of the solid for the hydrogen molecules, whereby they are less condensed at the surface than molecules of other gases, so that a given amount of vapour of water in the gas makes a larger impurity in the surface hydrogen than in the surface layers of other gases, and, secondly, the small mass of the hydrogen molecule. Thus we can give the following general sketch of the effect of the presence of water-vapour in a gas on the surface phenomena which we are treating of at present. Water-vapour appears almost to dissolve in solid glass, forming a union which also retains some free water-vapour at the surface; thus a vibrating solid surface would practically carry such a layer of water-vapour as part of itself, and thus the layer would introduce error into the slipping of the gas, the amount of which for the ordinary gases is small, but becomes important with hydrogen for the reasons already stated. Now if the water-vapour is mostly gathered at the surface it cannot affect the general viscosity of the gas, but only the slipping and the deflecting force. In the case of hydrogen the effect of the water-vapour seems to be to shorten the mean free path by an amount which is nearly constant, and, moreover, in the case of slipping alters the constant $a$ in the relation $\xi=a \lambda$ to $1 / 3$ of its usual value, so efficient is the layer of massive water molecules in sweeping the light hydrogen molecules with it. The curious result that the shortening of the free path of hydrogen near a solid surface by the watervapour should be independent or nearly independent of the pressure invites inquiry, but we can hardly delay any longer here on this hydrogen episode.

Resuming the study of the pressure-gauges, we will now calculate the pressures in Crookes's highest hydrogen vacua I 2 
according to the equations

$$
p\left(\frac{\mathrm{I}-\mu}{l-\mu}-1+\cdot 03\right)=2 a \lambda_{0} p_{0} / \mathrm{D}, . .
$$

with

$$
\mathrm{L}=\cdot 0499, \mu=\cdot 004 \text {, and } 2 a \lambda_{0} p_{0} / \mathrm{D}=10 \cdot 5,
$$

and deflecting force $=c^{\prime} /\left\{\mathrm{A}^{\prime \prime} p /(1-\alpha p)+\mathrm{B}^{\prime \prime \prime}+1 / p\right\}$, .

with $\quad c^{\prime}=4 \cdot 16, \mathrm{~A}^{\prime \prime}=\cdot 0006, \quad x=\cdot 0016, \mathrm{~B}^{\prime \prime \prime}=\cdot 01$.

$\begin{array}{lllllllllllll}10^{4} \log \text {. dec. } \ldots & 324 & 304 & 270 & 253 & 232 & 214 & 191 & 172 & 169 & 157 & 130 & 118\end{array}$

$\begin{array}{lllllllllllll}\text { def. for......... } & 49 & 45 & 37 & 31 & 29 & 26 & 15 & 10 & 9 & 7 & 3 & 2\end{array}$

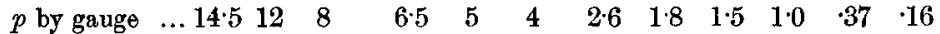

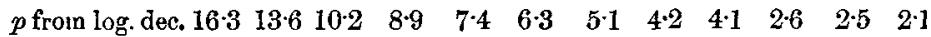

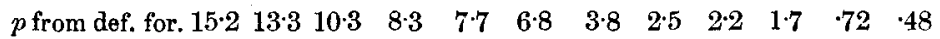

$$
\begin{array}{llllllllllll}
1.1 & 1.0 & 1.0 & 1.1 & 1.0 & .9 & 1.3 & 1.7 & 1.9 & 1.5 & 3.5 & 4.4
\end{array}
$$

The numbers in the last row are the ratios of the pressures given by Crookes's radiometer when used as a viscometer gauge and as a transpiration gauge, and they show that they agree with one another down to a pressure of abont $5 / 10^{6}$ atmo as given by the viscometer gauge, although at that pressure they have become 50 per cent. larger than the pressure given by M'Leod's gauge. Then, just as in the case of air, we find the value of the ratio rise suddenly to be nearly 2 instead of 1 , and the same explanation holds for the fact here as for air, namely that the mean free path is getting to be about as large as the radius of the bulb. Now as the mean free path in hydrogen is about double that in air, the pressure at which the change in the ratio occurs with hydrogen ought to be about double that with air, and as the change occurs at pressures about $5 / 10^{6}$ atmo and $2 \cdot 5 / 10^{6}$ as measured by the viscometer gauge, the theoretical condition is realized in the experimental results.

According to the viscometer gauge the lowest pressure is 13 times as great as that given by the $\mathrm{M}^{\prime}$ Leod gauge, a result which shows how useful the control of the different gauges by one another will be in measurements of the highest vacua. The change in ratio which we have found both with hydrogen and air seems at first sight to disqualify the transpiration gauge, but it is to be remembered that the magnitude of the change is the result of the unsuitableness of Crookes's torsion radiometer to serve as a type of the ideal transpiration gauge, which, if designed on the lines explained a few pages back, would be liable to only a trifling change of ratio, which could be reduced to zero if steps were taken to bring the whole 
inner surface of each of the two chambers connected by the annular space to a uniform temperature.

We will now briefly diseuss the performances of the two ganges with $\mathrm{CO}_{2}, \mathrm{CO}$, and $\mathrm{N}_{2}$ by means of Crookes's measurements of log. dec. and deflecting force, for using the latter of which the appropriate values of $e^{\prime}, \mathrm{A}^{\prime \prime}$, and $\mathrm{B}^{\prime \prime \prime}$ are given in "Thermal Transpiration and Radiometer Motion ;" for using Crookes's values of the log. dec. we have the following:-

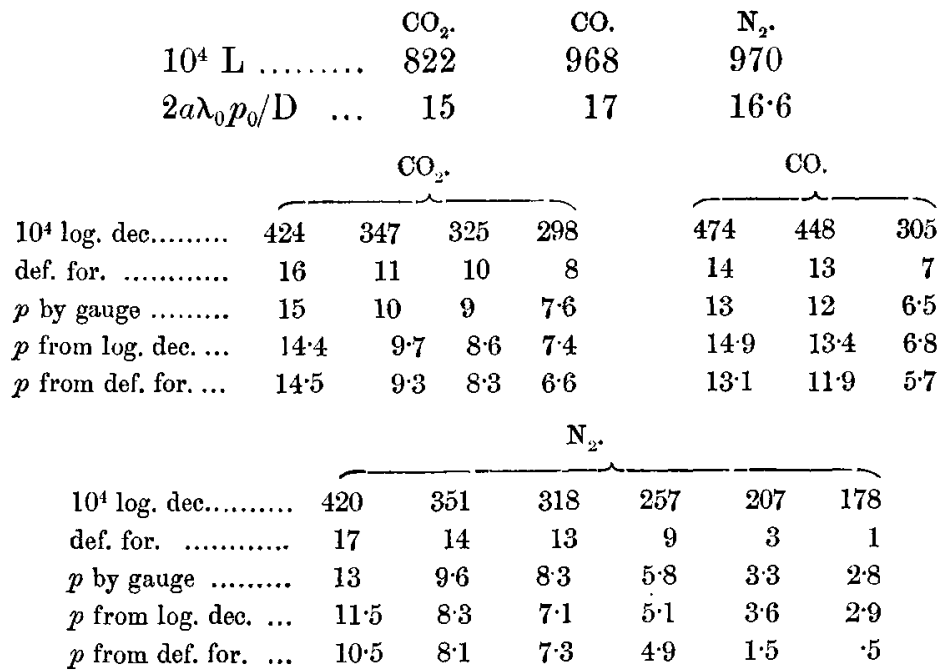

In the case of $\mathrm{N}_{2}$ the pressure from the log. dec. and that from deflecting force agree down to about $5 / 10^{6}$ atmo, although systematically less than those by the $M^{\prime}$ Leod gauge and Boyle's law; but at the two lowest pressures a great difference appears, which is too large for the same explanation as applied to the change of ratio with air and $\mathrm{H}_{2}$; in comparison with the deflecting force in air that in $\mathrm{N}_{2}$ dies away in a very sudden manner-for example, while at $3 / 10^{6}$ atmo the deflecting force in air is about 10 , in $\mathrm{N}_{2}$ it is only 1 , a very remarkable difference, calling for further experimental inquiry.

Crookes's measurements for oxygen, being of special importance, will be discussed separately in my next paper. The fairly good agreement which we have found between the indications of the M'Leod gauge at low densities and those of the viscometer gauge and the transpiration gauge furnish an indirect proof that there can be no surface condensation which suffices with the gases discussed to produce any appre- 
ciable departure from Boyle's law ; for if there were, there could not be a constant relation between density as found by the M'Leod gauge' and by the other two; but as to departure from Boyle's law due to other causes than surface condensation the agreement tells nothing, as it simply means an agreement in three measurements of density. But it is of some importance to learn that surface condensation produces no appreciable effect, even by way of an apparent departure from Boyle's law in rare gases (see "Boyle's Law at very Low Pressures") ; of course it is not implied that there is no surface condensation, for, on the contrary, its nature and amount have been calculated in "Boyle's Law at very Low Pressures," but in ordinary vessels the mass condensed is a small constant fraction (for a given gas) of the total mass in the vessel, and produces no effect on Boyle's law to the degree of accuracy of measurement.hitherto attained.

The trace of water-vapour which was the probable cause of the abnormalities discussed in connexion with hydrogen is also the probable cause of the failure of Crookes's M'Leod gauge at pressures near and below $1 / 10^{6}$ atmo both with hydrogen and air. As at the lowest pressure in air the $M^{\prime}$ Leod gange gave a measurement of the pressure only 1/26 of that of the viscometer gauge, it follows that $25 / 26$ of the gas in the highest vacuum would appear to bave been $\mathrm{H}_{2} \mathrm{O}$, which, when the gas is compressed into the volume-tube of the $\mathbf{M}^{\prime}$ Leod gauge, simply recombines with the glass again; if this is so, then the pressures calculated from the log. dec. and from the deflecting force both for air and hydrogen are inaccurate, as they ought to be calculated with the values of the parameters for $\mathrm{H}_{2} \mathrm{O}$ instead of those for air and $\mathrm{H}_{2}$. In fact, if at the highest vacua in both air and $\mathrm{H}_{2}$ water-vapour is the main constituent, then $l$, the log. dec., ought to have the same value both for air and $\mathrm{H}_{2}$ at the same erroneous values of the pressure given by the M'Leod gauge. The following little table furnishes a comparison of the log. decs.:-

$\begin{array}{ccccc}\text { apparent } p \ldots & 1 \cdot 3 & 1 \cdot 0 & \cdot 37 & \cdot 16 \\ 10^{4} l \text { for air... } & 175 & 161 & 126 & 115 \\ 10^{4} l \text { for } \mathrm{H}_{2} \ldots & 164 & 157 & 130 & 118\end{array}$

This shows that our supposition as to water's forming the chief ingredient of Crookes's highest vacua is probably true, and therefore the pressures which we previously obtained from the viscometer and transpiration gauges at the highest vacua are erroneous, being calculated for air and $\mathrm{H}_{2}$ instead of for $\mathrm{H}_{2} \mathrm{O}$, but obviously the amount of their error cannot 
attain to that of the indications of the M'Leod gauge. Thus there is strong evidence that in the investigations of highly rarefied gases in glass vessels there is always an unremoved trace of water which exercises a perturbing influence increasing with the rarefaction, and becoming serious in Crookes's experiments at about a millionth of an atmo. In investigating the highest vacua it would seem to be desirable to dispense with glass or to ascertain if glass can be obtained which does not exercise a special "dissolving" action on water-vapour.

Melbourne, August 1896.

XV. On the Relation of the Physical Properties of Aqueous Solutions to their state of Tonization. By Prof. J. G. MAC. Gregor, Dalhousie College, Halifax, N.'S.*

[Concluded from p. 55.]

Relative Values of a Property for a Mixture and for its Constituents. "Corresponding" Solutions.

$A$ change of ionization in general occurs on mixing two A solutions, it follows from (3) and (4) that the value of a property for a mixture of two solutions having one common ion will differ from the volume-mean, $\left(v_{1} \mathrm{P}_{1}+v_{2} \mathrm{P}_{2}\right) /\left(v_{1}+v_{2}\right)$, of its values for the constituents by the amount

$$
\left(l_{1}-k_{1}\right) \frac{n_{1} v_{1}}{v_{1}+v_{2}}\left(\alpha_{1}^{\prime}-\alpha_{1}\right)+\left(l_{2}-k_{2}\right) \frac{n_{2} v_{2}}{v_{1}+v_{2}}\left(\alpha_{2}^{\prime}-\alpha_{2}\right) .
$$

The name of "corresponding" solutions has been given to solntions for which this quantity vanishes. In general it will obviously have a value, though that value may be small.

In most cases this conclusion is borne out by experience. But Rother has concluded from his observations that, in the case of surface-tension, throughout a wide range of concentration, solutions of all concentrations are "corresponding." Were this the case it would throw serious doubt on the possibility of expressing surface-tension in terms of state of ionization. If, however, with the aid of the constants for surface-tension determined above, we compute, in the case of Sodium and Potassium Chlorides, the difference between the value for a mixture and the volume-mean of the values for its constituents, we find it to be beyond the limit of Rother's power of observation. Thus, in the case of his first mixture calculated above, the difference amounts to only $0 \cdot 0_{3} 15$. His

* Communicated by the Author. 